\title{
New Paradigms for the Old Question: Challenging the Expectation Rule Held by Risky Decision-Making Theories
}

\author{
Lei Zhou, ${ }^{1,2,3}$ Yang-Yang Zhang, ${ }^{3,2,4}$ Shu Li, ${ }^{2,3,1}$ and Zhu-Yuan Liang ${ }^{2,3}$ \\ ${ }^{1}$ Management School, Jinan University, Guangzhou, China \\ ${ }^{2}$ CAS Key Laboratory of Behavioral Science, Institute of Psychology, Beijing, China \\ ${ }^{3}$ Department of Psychology, University of Chinese Academy of Sciences, Beijing, China \\ ${ }^{4}$ School of Psychology, Shaanxi Normal University, Xi'an, China
}

\begin{abstract}
n risky decision making, whether decision makers follow an expectation rule as hypothesised by mainstream theories is a compelling question. To tackle this question and enrich our knowledge of the underlying mechanism of risky decision making, we developed a series of new experimental paradigms that directly examined the computation processes to systematically investigate the process of risky decision making and explore the boundary condition of expectation rule over the course of a decade. In this article, we introduce these methods and review behavioural, eye-tracking, event-related potential, and functional magnetic resonance imaging studies that employed these methods. Results of these studies consistently showed that decision makers in the single-application condition did not perform the weighting and summing process assumed by the expectation rule. Moreover, decision makers were inclined to adopt a non-compensatory strategy, such as a heuristic one, in risky decision making. Furthermore, results indicated that the expectation rule was only applicable for conditions that involved decisions applied to numerous events (multiple applications) or to people (everyone). The findings indicated that using an index based on expected value to prescribe human risk preferences appears to be an artificial or false index of risk preference, and emphasised a new methodological direction for risky decision-making research.
\end{abstract}

Keywords: risky decision making, process testing, compensatory rule, non-compensatory rule

Decision making under risk is vital to human survival and development. Risky choices, such as investing or eating genetically modified foods, are common in everyday life. Determining how people make risky choices is a compelling question for scientists. For theoretical studies, a debate on whether decision making under risk is based on a compensatory or non-compensatory process is ongoing. Mainstream theories of decision making under risk claim that risky choices are based on a compensatory expectation-maximisation process. When making a risky choice, people weigh each possible outcome by its probability and then sum all the risky outcomes to assign an overall value (expectation) to each option. Then, they select the option that offers the highest overall expectation (Glöckner \& Herbold, 2011; Kahneman \& Tversky, 1979; Tversky \& Kahneman, 1992). However, models that follow the non-compensatory rule and assume that people rely on only one (or a few) key dimension(s) rather than integrating information from all dimensions of an option to reach a decision are available (Brandstätter, Gigerenzer, \& Hertwig, 2006; S. Li, 2004a; Thorngate, 1980). For example, the equate-to-differentiate model suggests that when making risky choices, people seek to 'equate' the less significant differences between options in either the best or worst possible payoff dimensions, and rely on only the large one-dimensional difference to be differentiated as the determinant of the final choice (S. Li, 2004a; S. Li \& Xie, 2006).

As the research focus has shifted from understanding what people choose to how they decide (SchulteMecklenbeck, Kühberger, \& Hutzler, 2017), methods for examining risky decision-making models have been developed from outcome-based or goodness-of-fit techniques (Liang, Xu, Rao, Jiang, \& Li, 2012) to process-tracing techniques. Outcome-based techniques compare choices of individuals with the predicted choices of certain models (Brandstätter \& Gussmack, 2012; Gigerenzer, Hertwig, \& Pachur, 2011; Pachur, Hertwig, Gigerenzer, \&

Address for correspondence: Drs Shu Li or Zhu-Yuan Liang, Institute of Psychology, Chinese Academy of Sciences, Beijing 100101, China. E-mail: lishu@psych.ac.cn; liangzy@psych.ac.cn 
Brandstätter, 2013). However, stating a preference fails to serve as a true process measure because it provides no information regarding the necessary steps that lead to the preference (Schulte-Mecklenbeck, Kühberger, \& Ranyard, 2010). Concrete and convincing evidence based on psychological processes is necessary to elucidate this process and further our understanding of the underlying mechanisms of human decision making. Consequently, researchers have begun to examine the underlying processes of risky choice. Process data are richer than inputoutput data and provide important evidence for explanatory mechanisms (Schulte-Mecklenbeck et al., 2010). Various technologies used to study information processing of people during decision making include behavioural experiments, functional magnetic resonance imaging (fMRI), event-related potential (ERP), mouse tracking, and eyetracking (Dshemuchadse, Scherbaum, \& Goschke, 2013; Glöckner \& Herbold, 2011; Rao, Li, Jiang, \& Zhou, 2012; Rao et al., 2013; Rao et al., 2011; Scarpa, Zanoli, Bruschi, \& Naspetti, 2013; Su et al., 2013). However, a few researchers have decomposed the decision-making process and directly assessed the computational steps assumed by the expectation rule by weighting, summing, and maximising the overall values.

Despite the uneven representation of judgment and decision making worldwide and at its traditional study centre in Israel (Baron, 2008), researchers from the Pacific Rim have provided theoretical and applied contributions of risky decision-making studies. The research conducted in the Pacific Rim has shed light on unique cultural and regional characteristics of risky decision making. Historically, most of the studies in the earlier stage have endorsed compensatory models. For example, Quiggin (1982) from Australia was the first to find an appropriate model, namely the rank-dependent utility model, for the utilisation of transformed probabilities in risky choices (Fennema \& Wakker, 1994). Further, Grant and Kajii (1998), from the Australian National University, provided an axiomatisation for the representation of risky choice that is a special case of rank-dependent expected utility called AUSI (pronounced 'ozzie') expected utility. Similar to this trend, a small but growing number of researchers have begun to explore the decision-making anomalies that challenged the compensatory models, or they have developed non-compensatory models. For example, Takemura (1994), from Japan, proposed the contingent focus model to explain the framing effect in risky decision making. In a subsequent study, Takemura and Fujii (1999) applied the model to a psychometric metaanalysis of subject responses in experiments on the Asian disease problem (Tversky \& Kahneman, 1981) and found that the results were compatible with the predictions of the model. Moreover, S. Li and colleages (2016) developed a coherent 'equate-to-differentiate' account for the most reported anomalies and perplexing phenomena in risky decision making. The anomalies and phenomena include the framing effect on the Asian disease problem
(S. Li, 1998; S. Li \& Adams, 1995; S. Li \& Xie, 2006), the graph framing effect (Y. Sun, Bonini, \& Su, 2012), the Allais paradox (S. Li, 1993, 1994a), the certainty effect (S. Li, 1995), the reflect effect (a four-fold pattern of risk attitudes; S. Li, 1993, 2004b), preference reversal (S. Li, 1994b, 2006), single- versus multiple-play discrepancy identical to the symbol (S. Li, 2003), and the disjunction effect in prisoner dilemma games (S. Li \& Taplin, 2002; S. Li, Taplin, \& Zhang, 2007). Baron (2016, p. 4) noted that the equate-to-differentiate model is 'an interesting theory that can account for numerous results'. The model particularly emphasises our tendency to reduce decisions to 'dominance structure', in which real conflicts and trade-offs disappear (Baron, 2016, p. 4). Apart from these theoretical studies, applied studies have focused on hazardous risk management due to the prevalence of natural hazardous circumstances in the Pacific Rim region (Paton, 2009). These applied studies include the profactors of hazard preparedness (Paton et al., 2009; Sagala, Okada, \& Paton, 2009), survivor response to a disastrous experience (Nalipay \& Mordeno, 2016; Seiuli, Nikora, Te Awekotuku, \& Hodgetts, 2016), effect of earthquake exposure on mood (Aslam \& Tariq, 2010), and intervention evaluation aimed at teaching adaptive coping skills for disaster survivors (Hechanova, Waelde, \& Ramos, 2016).

As one collaborative part of the preceding studies mentioned in the Pacific Rim, we developed a series of new paradigms to systematically investigate risky decision making over the past decade from a theoretical perspective. Part of the paradigms were designed to explore whether the risk-decision process follows the expectation rule by directly examining the hypothesised computation processes. Other paradigms focused on exploring the boundary condition of the expectation rule, that is, exploring the context under which the rule will work. Based on these paradigms, we conducted behavioural and neurological experiments using fMRI, ERP, eye-tracking, and behavioural experiments. These studies provided convergent evidence regarding the extent of risky decision making based on weighting and summing processes. These paradigms and studies are summarised in Table 1.

\section{Methods for Investigating Decision Process}

Theories are developed to answer 'what,' 'how,' and 'why' questions (Busse, Kach, \& Wagner, 2017). The first part of our work focused on the 'what' question and directly investigated the process of risky decision making, especially the weighting and summing process. In these methods, participants completed risky decision making and baseline tasks. In the baseline task, participants consciously performed a weighting and summing process. Thus, the differences in decision process between a risky decisionmaking task and the baselines may reveal whether participants perform the weighting and summing process assumed by the expectation rule. 
Table 1

Paradigms for the Examination of Risky Decision Making

\begin{tabular}{|c|c|c|c|c|}
\hline Paradigm & Techniques & $\begin{array}{l}\text { Feature-independent } \\
\text { and moderator/ } \\
\text { mediator variables }\end{array}$ & $\begin{array}{l}\text { Feature-dependent } \\
\text { measures }\end{array}$ & Authors (year) \\
\hline \multicolumn{5}{|c|}{ Methods of investigating decision process } \\
\hline \multirow[t]{2}{*}{$\begin{array}{l}\text { Proportion versus } \\
\text { probability method }\end{array}$} & $\begin{array}{l}\text { Behavioural } \\
\text { experiment }\end{array}$ & $\begin{array}{l}\text { Computational difficulty; sensation } \\
\text { seeking; numerical capability; } \\
\text { arithmetic capability }\end{array}$ & $\begin{array}{l}\text { Response time; proportion of } \\
\text { choice }\end{array}$ & Liang et al. (2012) \\
\hline & Eye-tracking & Computational difficulty & $\begin{array}{l}\text { SM value; similarity score of } \\
\text { the scanpath, typical trial }\end{array}$ & $\begin{array}{l}\text { Su et al. (2013); Zhou } \\
\text { et al. (2016) }\end{array}$ \\
\hline \multirow[t]{3}{*}{$\begin{array}{l}\text { Judgment-based versus } \\
\text { preferential choice } \\
\text { method }\end{array}$} & fMRI & Decision conflicts & $\begin{array}{l}\text { Activity of conflict-related } \\
\text { regions; functional } \\
\text { connectivity strength }\end{array}$ & Rao et al. $(2011,2012)$ \\
\hline & ERP & $\begin{array}{l}\text { Difference in the minimum outcome } \\
\text { dimension between two options; } \\
\text { computational difficulty }\end{array}$ & $\begin{array}{l}\text { P300; slow wave component } \\
\text { (500-800 ms) }\end{array}$ & Rao et al. (2013) \\
\hline & Eye-tracking & Decision rules & $\begin{array}{l}\text { Dwell time; fixation counts; } \\
\text { number of saccades; } \\
\text { information search patterns }\end{array}$ & Wang and Li (2012) \\
\hline $\begin{array}{l}\text { Outcome-matched versus } \\
\text { outcome-crossed } \\
\text { method }\end{array}$ & Eye-tracking & $\begin{array}{l}\text { Position of the best/worst outcomes } \\
\text { (matched/crossed) }\end{array}$ & $\begin{array}{l}\text { Attribute-based saccades } \\
\text { between best/worst possible } \\
\text { outcomes; similarity score of } \\
\text { the scanpath, typical trial }\end{array}$ & $\begin{array}{l}\text { Wang and Li (2012); } \\
\text { Zhou et al. (2016) }\end{array}$ \\
\hline $\begin{array}{l}\text { Imposed-rule versus } \\
\text { self-rule tasks }\end{array}$ & $\begin{array}{l}\text { Behavioural } \\
\text { experiment }\end{array}$ & $\begin{array}{l}\text { Decision rules (self-rule vs. imposed } \\
\text { rule) }\end{array}$ & $\begin{array}{l}\text { Evaluation of emotions and } \\
\text { acceptance }\end{array}$ & Rao et al. (2009) \\
\hline \multicolumn{5}{|c|}{ Methods of examining boundary condition } \\
\hline $\begin{array}{l}\text { Single-application versus } \\
\text { multiple-application } \\
\text { method }\end{array}$ & Eye-tracking & Computational difficulty & $\begin{array}{l}\text { SM value; choice; proportion of } \\
\text { inter/intra-dimensional } \\
\text { saccades; similarity score of } \\
\text { scanpath; typical trial }\end{array}$ & $\begin{array}{l}\text { Su et al. (2013); H.Y. } \\
\text { Sun et al. (2013); } \\
\text { Zhou et al. (2016) }\end{array}$ \\
\hline $\begin{array}{l}\text { Decision for everyone } \\
\text { versus every one method }\end{array}$ & Eye-tracking & $\begin{array}{l}\text { EV difference; outcome difference; } \\
\text { percentage of total information } \\
\text { searched; mean fixation duration; } \\
\text { SM value; proportion of the saccades } \\
\text { between the two best outcomes }\end{array}$ & $\begin{array}{l}\text { Similarity score of the } \\
\text { scanpath; percentage of } \\
\text { total information searched; } \\
\text { mean fixation duration; SM } \\
\text { value; proportion of the } \\
\text { saccades between the two } \\
\text { best outcomes }\end{array}$ & Liu et al. (2017) \\
\hline
\end{tabular}

Note: $\mathrm{fMRI}=$ functional magnetic resonance imaging, $\mathrm{ERP}=$ event-related potential, $\mathrm{SM}=$ search measure

\section{Proportion Versus Probability Method}

This method was designed to examine the weighting process in risky decision making. Participants performed proportion and probability tasks in this method. Materials in the two tasks were visually identical to the symbol ' $\mathrm{x} \%$,' thereby indicating that 'you will obtain/lose an $\mathrm{x} \%$ proportion of this payoff' in the proportion task and 'you will have an $\mathrm{x} \%$ probability of obtaining/losing this payoff' in the probability task (Figure 1). In the proportion task, participants were required to select between riskless options that involved several partially available payoffs. Meanwhile, the participants were required to select between risky options that involved several probabilistic payoffs in the probability task. In the proportion task, a natural mental arithmetic process is consistent with the weighting and summing processes predicted by compensatory models. Payoffs in each option are first weighted by their respective proportions and then summed to obtain the overall payoff that determines the final choice. If individuals engage in weighting and summing processes when making risky choices, then their performance in the probability task should be similar to their performance in the proportion task. Thus, differences in performance would suggest that risky decision making is not based on weighting and summing processes.

Behavioural and eye-tracking studies have shown the proportion versus probability method to be a valid assessment of the extent to which the expectation rule operates in risky choices. Liang et al. (2012) conducted behavioural experiments using the proportion versus probability method and found that participants exhibited shorter response times in probability tasks compared with proportion tasks, and proportions of expected value-based choices were lower in probability tasks than in proportion tasks. Their study employed double dissociation in comparing the two types of tasks. The rationale underlying the hypothesis of double dissociation is as follows: if the same cognitive process $\mathrm{C}$ is involved in tasks $\mathrm{A}$ and $\mathrm{B}$, then $\mathrm{C}$ should influence $\mathrm{A}$ and $\mathrm{B}$. However, if cognitive process $\mathrm{C}$ is only involved in task $\mathrm{A}, \mathrm{C}$ should only influence task $\mathrm{A}$ and not affect task $\mathrm{B}$. The study results demonstrated that arithmetic capability, which influences weighting and summing processes, moderated performance in the proportion tasks but not in the probability tasks. By contrast, numerical capability and the sensation-seeking trait, which influence risky decision making, moderated 


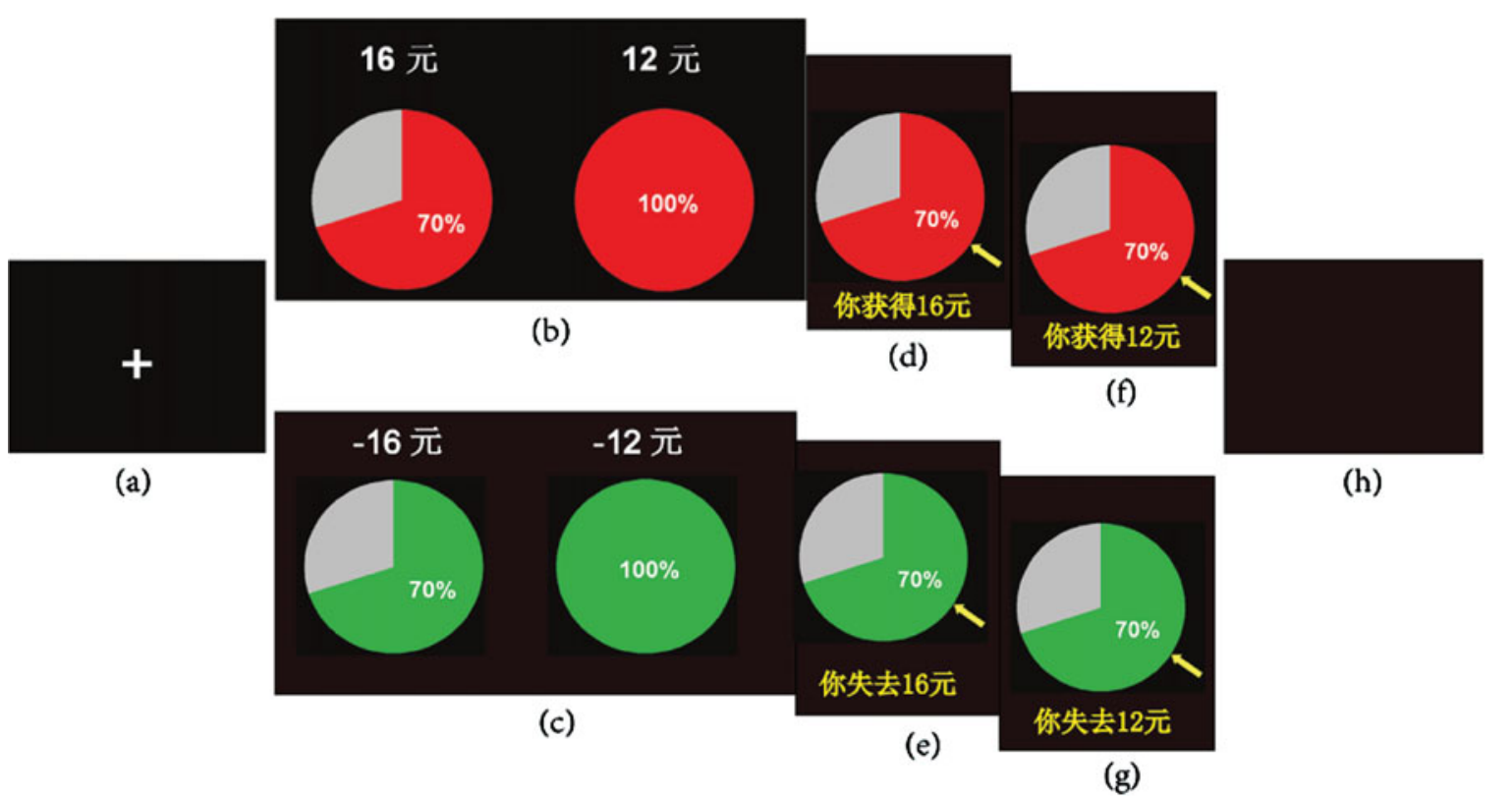

\section{Figure 1}

Trial structure. (a) fixation; (b) and (c) experimental material in gain/loss domain; (d) and (e) feedback of choice for proportion task in gain/loss domain; (f) and ( $\mathrm{g}$ ) feedback of choice for probability task in gain/loss domain; (h) blank. In the proportion task, participants select between two riskless options of obtaining (top panel) or losing (lower panel) an $\mathrm{x} \%$ proportion of a payoff; in the probability task, participants select between two risky options with an $\mathrm{x} \%$ probability of obtaining (top panel) or losing (lower panel) a payoff (Liang et al., 2012).

performance in the probability tasks but not in the proportion tasks. These results demonstrated that weighting and summing processes were not involved in the probability task.

Su et al. (2013) employed this method in an eyetracking study and found that eye movements of participants were significantly different in the probability task compared to those of the proportion task. Specifically, they found significantly different information search patterns in the two tasks based on the alternative-based versus dimension-based search measure (SM; Böckenholt \& Hynan, 1994) of the two tasks. Attribute-based saccades, which are consistent with the prediction of weighting and summing processes, were observed to predominate in the proportion task. However, dimension-based saccades, which are consistent with the predictions of heuristic processes, were observed to predominate in the probability task. Based on these results, the researchers concluded that different processes governed information search and processing of participants in the proportion and probability tasks, thereby indicating the absence of weighting and summing processes when people make risky choices. Zhou et al. (2016) analysed the published data from the eyetracking study of Su et al. (2013) using scanpath analysis. Compared with traditional eye-tracking analysis methods, scanpath analysis provides a global view of the decisionprocess analysis. The similarity scores between scanpaths indicate the extent to which the scanpaths of different tasks are similar (X. Li, Logan, \& Zbrodoff, 2010). Zhou et al. (2016) also developed a new method for identifying a typical trial and provided a visualisation of the decision process. They found that the internal consistency of the scanpath pattern was higher in the proportion task than in the probability task. The researchers also found significant differences between the scanpath patterns in the proportion and probability tasks. The scanpath in a typical trial of the proportion task exhibited a pattern that was consistent with weighting and summing processes. However, this pattern was not observed in the probability task.

\section{Judgment-Based Versus Preferential Choice Method}

This method was designed to assess the extent to which preferential choice of an individual involved a compensatory process by examining weighting, summing, and expectation-maximisation processes. In this method, the rules followed by participants in making risky choices were different in the judgment-based and preferential choice tasks. The judgment-based choice task, which requires participants to use the certainty equivalent method, implicitly forced participants to employ a compensatory process. However, the preferential choice task did not explicitly require participants to perform a compensatory or non-compensatory process. Contrasting judgment-based choice with preferential choice enabled us to determine whether preferential choice of an individual could be characterised as a compensatory process.

In their fMRI and ERP studies, Rao, Li et al. (2012), Rao, Lui et al. (2013), and Rao, Zhou et al. (2011) focused on the neural basis of conflict monitoring by comparing a judgment-based choice task with a preferential choice task. Their series of studies provided neurological evidence that preferential choice cannot be solely characterised as a 
compensatory process. The first fMRI study (Rao et al., 2011) examined the extent to which risky choices involve a compensatory process from the perspective of decision conflict. The activity in conflict-related brain regions during the two tasks was compared to assess whether the intensity of inner conflict was less pronounced in the judgment-based choice task than in the preferential choice task. The primary difference between compensatory and non-compensatory processes appeared to be associated with differences in conflict resolutions between binary alternatives (Hogarth, 1987). In a compensatory process, a strong conflict is induced by maximisation when the difference between the overall values or utilities of two alternatives is small. In a non-compensatory process, a strong conflict is related to large intra-dimensional differences between the two alternatives in payoff and probability. The results in the preferential task indicated that conflictrelated regions were sensitive to the intra-dimensional difference (i.e., difference between the probability/payoff dimensions of two risky options). The conflict-related regions in the judgment-based task were sensitive to the unidimensional difference (i.e., difference between the overall values/utilities of two risky options). Moreover, researchers also found that activation in conflict-related brain regions increased during the preferential choice task compared to the judgment-based choice task. Overall, the results of this study indicated that preferential choice cannot be characterised as a compensatory process that maximises the overall value of a prospect in a conflict solution.

Rao et al. (2012) also obtained neurological evidence from functional connectivity analysis for the judgmentbased and preferential choice methods. They classified brain regions into probability and payoff networks based on the functional connectivity patterns between regions. In these regions, activity was detected during both types of decision-making tasks. Moreover, the probability and payoff networks were found to exhibit stronger connectivity strength in the judgment-based choice task compared to the preferential choice task. These results indicated that the weighting process uniformly predicted by mainstream theory is unnecessary during preferential choice.

Rao et al. (2013) used ERPs to investigate the judgment-based and preferential choice tasks. The study employed the following double dissociation rationale: if the difference in magnitude in the minimum outcome dimension (which affects risky decision making) only influences the decision process in the preferential choice task, whereas computational difficulty (which influences weighting and summing processes) only affects the judgment-based choice task, then preferential choice does not appear to be based on a compensatory process. The researchers also found that the $\mathrm{P} 300$ wave reflected the outcome of stimulus evaluation, and slow wave components were usually linked to the mental processes essential to calculation prior to obtaining the arithmetic operation result (Rao et al., 2013). The ERP data collected in the two tasks indicated a hypothesised double dissociation. In the preferential choice task, P300 was sensitive to the difference magnitude in the minimum outcome dimension but insensitive to computational difficulty, whereas the slow wave component (500-800 ms) was sensitive to computational difficulty in the judgment-based choice task. These results indicated that the predicted mental arithmetic process occurred in the judgment-based choice task but not in the preferential choice task. Given that computational difficulty is a key feature of mental arithmetic processes, the ERP data suggest that the brain mechanism underlying risky choice is not based on a compensatory process.

In their eye-tracking study, Wang and $\mathrm{Li}$ (2012) compared eye movement patterns in judgment-based and preferential choice tasks and found that dwell time, fixation count, and number of saccades significantly differed in the two types of tasks. Their results also indicated that option-based information searches were predominant in the judgment-based choice task, whereas attribute-based information searches were predominant in the preferential choice task. These findings conflict with the predictions of the compensatory model.

\section{Outcome-Matched Versus Outcome-Crossed Method}

This method assessed the summing process by comparing outcome-matched and outcome-crossed stimuli presentations. Participants were presented with pairs of options in which each option was associated with the following two outcomes: best and worst. As illustrated in Figure 2, the best and worst outcomes for each option were presented as either parallel (outcome-matched) or crossed (outcome-crossed). Wang and Li (2012) used this method in their eye-tracking study. They hypothesised that if risky choices were based on the expectation rule, then horizontal saccades would not be influenced by the format of stimuli presentation. However, if risky choices involved a non-compensatory strategy, such as the priority heuristic (Brandstätter et al., 2006) or equate-to-differentiate approach (S. Li, 2004a), then attribute-based saccades between best and worst possible outcomes should occur in parallel in the outcome-matched stimuli presentation and crossed in the outcome-crossed stimuli presentation.

The eye-tracking study of Wang and Li (2012) supported the preceding hypothesis and suggested that risky decision making is not based on a compensatory strategy. The scanpath analysis indicated that the scanpath patterns of the outcome-matched and outcome-crossed tasks significantly differed (Zhou et al., 2016). The scanpath patterns observed in typical trials were more consistent with the predictions of the equate-to-differentiate approach (S. Li, 2004a; Li \& Xie, 2006) than those of the compensatory expectation rule.

\section{Imposed Rule Versus Self-Rule Method}

This method was developed to examine emotion and acceptance after decision making when individuals make decisions according to their self-rule (i.e., spontaneously 
Outcome-crossed presentation

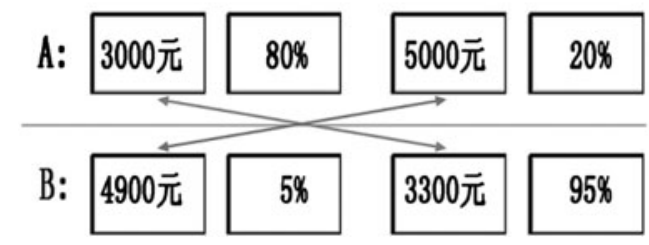

Outcome-matched presentation

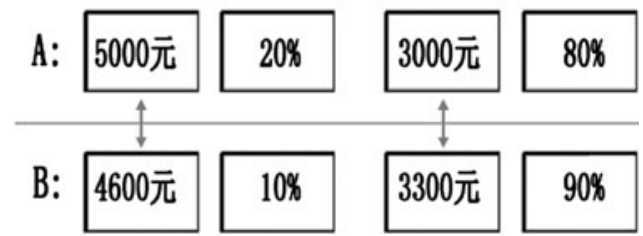

\section{Figure 2}

Illustration of outcome-crossed versus outcome-matched presentations. Left panel (outcome-crossed presentation): The best/worst possible outcomes of A (CNY $5000 /$ CNY 3000) and the best/worst possible outcomes of B (CNY 4900/CNY 3300) were presented crossed, as indicated by the arrows. Right panel (outcome-matched presentation): The best/worst possible outcomes of A (CNY 5000/CNY 3000) and the best/worst possible outcomes of B (CNY 4900/CNY 3300) were presented in parallel, as indicated by the arrows (Zhou et al., 2016).

generated by the decision maker) and imposed rule (i.e., $\mathrm{EV}$ rule, which is defined based on decision theory). Logically, individuals would experience positive emotions and exhibit high levels of acceptance for decisions based on their own 'true' rule. Therefore, consistency between imposed rule and self-rule will lead to the reported acceptance and positive emotion by individuals after decision making. Following the logic, researchers can compare the compensatory and non-compensatory rule with the rule of decision makers and infer which rule is closer to the true rule of decision makers according to differences of individual acceptance and positive emotion to those rules.

In the study by Rao, Liang, and $\mathrm{Li}$ (2009), participants were instructed to make risky choices based on their own rule or imposed rules. Rao et al. employed the rules hypothesised by expected value theory and equate-todifferentiate theory as two imposed rules. In the self-rule condition, participants rated their emotions after making choices based on their own rule. In the imposed-rule condition, participants rated their emotions and acceptance of the imposed rule after making choices based on the imposed rule. The results revealed that participants reported higher levels of acceptance and positive emotions when choices were based on their own rules than when their own rule and the imposed rule produced the same decisions. Notably, the 'true' rule of decision makers was more likely to be consistent with the equate-to-differentiate rule than with the expected value rule. Their findings suggest that the expectation rule might fail to capture the emotion and acceptance after risky choice decision making of individuals.

\section{Methods of Examining Boundary Condition}

Distinct from theory development, the 'boundary condition' of theories refers to the 'who', 'where', and 'when' aspects of a theory to describe the limits of theory generalisability (Busse et al., 2017). Another part of our work focused on the 'when' aspects of risky decision-making theories and explored the boundary condition of the expectation rule by contextual factors, that is, defining under what context the expectation rule will work. We explored whether the expectation rule can be applicable for condi- tions that involved a single application or multiple applications or people (everyone).

\section{Single-Application Versus Multiple-Application Method}

This method was designed to examine weighting and summing processes in risky decision making, in which participants were asked to make risky choices in single- and multiple-application tasks. In each task, participants select between two options, in which at least one risky option involves a probability of obtaining or losing a payoff. In the single-application task, the selected risky option is only applied once; in the multiple-application task, the selected risky option is applied 100 times. Empirical research found that the behaviour of decision makers in multiple-application gambles is consistent with the predictions of compensatory theory (Colbert et al., 2009; DeKay et al., 2006; Keren, 1991; Klos, Weber, \& Weber, 2005; Langer \& Weber, 2001; S. Li, 2003; Redelmeier \& Tversky, 1992; Wedell \& Böckenholt, 1994). The deviation of people's actual decision behaviour from the predictions of expectation models might be influenced by differences between single- and multiple-application tasks. If singleand multiple-application tasks are significantly different, then the expectation rule is unlikely to govern risky choice in the single-application task.

Su et al. (2013) and H.Y. Sun, Rao, Zhou, and Li (2013) used eye-tracking to investigate single- and multipleapplication tasks. In the study of Su et al., participants were required to select between pairs of risky two-payoff monetary options (i.e., each option involved two risky outcomes) in both tasks. The option pairs were divided into two equal groups based on the level of difficulty of the total payoff computation of expectation value $(\mathrm{EV}$, high vs. low). Their study found that in the multipleapplication task, the compensatory models performed significantly better than the non-compensatory models in predicting choices, decision times, and eye movements of participants. Moreover, increased computational difficulty, which was expected to influence weighting and summing processes, affected performance only in the multiple-application task. Information acquisition in the multiple-application task generally took the form of 
alternative-based saccades and was consistent with weighting and summing processes. However, information acquisition in the single-application task generally took the form of dimension-based saccades, which is consistent with heuristic processes. These results suggest that an expectation-based index for risk preference prediction operates in multiple-play tasks but not in single-play tasks.

H.Y. Sun et al. (2013) compared single- and multipleapplication tasks by using behavioural and eye-tracking methods. In this study, participants performed single- and multiple-application tasks, each of which presented two scenarios. The first scenario was the Asian disease problem (Tversky \& Kahneman, 1981), and the second scenario was the food problem. Each scenario involved the following two emergency plans: the first plan produced a certain outcome, whereas the other produced a risky and high EV outcome. Different selections and eye movement patterns were found in the two tasks. Compared with the single-application task, more EV-based choices and interdimensional saccades and fewer intradimensional saccades were observed in the multiple-application task. Scanpath analysis indicated that scanpath patterns in the single- and multiple-application tasks significantly differed (Zhou et al., 2016). In the multiple-application tasks, the scanpath in the typical trial exhibited a pattern that was consistent with weighting and summing processes. However, this pattern was not observed in the single-application task.

\section{Decision for Everyone Versus Every One Method}

In their study, Liu et al. (2017) designed three risky choice tasks that comprised a decision-for-everyone (Deveryone) task, a decision-for-self multiple-play (Dmultiple) task, and a decision-for-self single-play (Dsingle) task to explore the boundary condition of EV rule. This task is a ramification of the single/multipleapplication task and aims to examine whether individuals apply the same strategy when making decisions for everyone (many people) or for the multiple-play condition when making decisions for themselves. In the $\mathrm{D}$-everyone task, decision makers select the more optimal option from the two options under the assumption that their choice will be a final decision for everyone facing the same choice. In the D-multiple task, decision makers select between the two options under the assumption that their choice will be applied 100 times. In the D-single task, decision makers select between two options under the assumption that their selection will be applied only once to themselves. If decision makers adopt the same strategy (i.e., the expectation strategy) in the D-everyone task and D-multiple task and a different strategy (i.e., a heuristic/non-expectation strategy) in the D-single task, then their performance on the D-everyone and D-multiple tasks should be similar and should contrast with that on the D-single task.

Liu et al. (2017) conducted an eye-tracking experiment using this task. They found that participants were more likely to follow an expectation strategy in the D-everyone task and the D-multiple task but were more likely to follow a heuristic/non-expectation strategy in the D-single task. These findings were based on behavioural and eyemovement results. The behavioural results indicated that participants in the D-everyone and D-multiple tasks were likely to select an option with higher EV as predicted by expectation rule choices than in the D-single task. Regarding the eye-movement data, the scanpath analysis revealed that the scanpath patterns in the $\mathrm{D}$-everyone and D-multiple tasks were similar to each other and different from those in the D-single task. Results from other eyetracking measures suggested that the depth of information acquisition and the complexity of information processing were lower in the $\mathrm{D}$-single task than in the $\mathrm{D}$-everyone and D-multiple tasks. Moreover, participants were more likely to use an alternative-based approach in the D-everyone and D-multiple tasks than in the D-single task. An interaction effect between task and EV difference showed that EV difference affected the mean fixation duration only in the D-everyone task. In addition, a mediation analysis revealed that the eye-tracking measures mediated the relationship between task and percentage of the EV-consistent choice. Overall, these results indicate that the expectation rule only works well for everyone (full set) and not for everyone (subset).

\section{Discussion and Conclusion}

In the last decade, we have developed a series of methods to directly examine risky decision-making models. The methods of investigating the decision process aim at investigating risky decision-making by comparing the process of a baseline task following a weighting and summing process, and a risky choice task. These studies revealed that participants do not perform the weighting and summing process assumed by the expectation rule and are more likely to adopt a non-compensatory strategy. The methods of examining boundary condition focus on exploring the boundary condition of expectation rule. These studies found that the expectation rule works for risky decisions that are applied numerous times (multiple application) or for people (everyone).

Compared with previous methods that rely on outcome-based techniques, our paradigms have provided several research contributions on risky decision making. First, we adapted paradigms that directly examined the computation processes of weighting, summing, and expectation-maximisation, which are assumed by the expectation rule. To the best of our knowledge, only a few previous studies achieved this goal. Considering that different decision models may predict the same outcome or preference despite potential differences in underlying processes, justifying decision models without examining the process is difficult (Johnson, Schulte-Mecklenbeck, \& Willemsen, 2008; Zhou et al., 2016). In addition, we can only determine which decision model works 
better when relying on outcome-based techniques by comparing different model candidates. However, if decision makers adopt multiple strategies assumed by different models, then distinguishing decision strategies using outcome-based techniques is difficult. A better approach for exploring such issues might be directly dependent on process data. Second, we directly compared information search and processing in risky decision making with information search and processing in a series of baseline tasks in which people naturally perform a deliberate calculation of the weighted sums. To a certain extent, simultaneously performing between-task comparisons enabled these paradigms to overcome the limitation on deducing hypotheses on the process data from the decision models that did not consider the relevant processes (Su et al., 2013). Third, we utilised a variety of process-tracing techniques, such as eye tracking, ERP, and fMRI, to provide convergent evidence to examine the expectation rule. Finally, the novel indexes used in the study provided global and direct measures of information search patterns. For example, the SM value is an index that quantifies the degree to which the search direction is alternative or dimension based (Böckenholt \& Hynan, 1994; Su et al., 2013). This measure enables the direct testing of hypotheses on the information search direction in risky decision making. Scanpath analysis, which focuses on the sequence of eye movements, provides spatiotemporal data on the spatial distribution of attention across a visual stimulus (Gbadamosi \& Zangemeister, 2001; Noton \& Stark, 1971; Underwood, Humphrey, \& Foulsham, 2008; Zhou et al., 2013). The pattern generated by the scanpath enables researchers to investigate the process of risky choice using a global measure. These indexes emphasise a methodological application and can be used not only in the field of decision making but also in other areas.

Our findings using these paradigms have important theoretical and practical implications. Theoretically, these studies suggest that using an EV-based index to prescribe human risk preferences appears to be an artificial or false index of risk preference and reveal the need to improve current theories that are based on the use of heuristic strategies. Practically, we indicate a future methodological direction by providing references for paradigm development and utilising novel indexes for the direct investigation the decision processes.

Notably, these methods could be improved by resolving certain limitations. The paradigms in this study only made assumptions on weighting and summing processes. Consequently, such paradigms could only be applied to the examination of decision models involving such processes. In addition, the evidence was insufficient for exploring rules that should be followed in risky decision making. Future studies could focus on varied decision processes and broaden the scope of decision models that could be examined. More importantly, except for falsification, future paradigms could also consider verification of the actual process that individuals follow in risk decision making.
In addition, participants in our studies were from a single race (i.e., Chinese), whereas the mainstream economic theory was dominantly built on participants of another single race (i.e., Caucasians). Owing to the ample findings that Asian cultures (e.g., Chinese) are more risk seeking than respondents in other cultures (e.g., in the United States; S. Li, 2001; Weber, Hsee, \& Sokolowska, 1998), the culture difference might confuse the results. Future studies should consider expanding their application to examine other decision processes of participants from multiple cultures and reveal the rules that individuals follow in risky choice.

In conclusion, this article reviewed paradigms of examining risky choice models developed for the following two aims: investigating the weighting and summing process assumed by the EV rule and exploring the boundary conditions by contextual factors of the EV rule. The results of the first cluster paradigms revealed that decision makers did not follow the EV rule in the single-application risky decision-making. By contrast, decision makers adopted a non-compensatory strategy, such as a heuristic one. The results of the second cluster paradigms showed that the EV rule was only applicable for conditions which involved decisions that applied numerous times (multiple application) or people (everyone).

\section{Acknowledgements}

We thank Hong-Zhi Liu for discussion. We thank LetPub (www.letpub.com) for its linguistic assistance during the preparation of this manuscript.

\section{References}

Aslam, N., \& Tariq, N. (2010). Trauma, depression, anxiety and stress among individuals living in earthquake affected and unaffected areas. Pakistan Journal of Psychological Research, 25, 131-148.

Baron, J. (2008). Foreword. In J. Baron, 思维与决策 (第四版) [Thinking and deciding (4th ed.)] ( S. Li \& Z.-Y. Liang, Trans.) (pp. V). Beijing, China: China Light Industry Press.

Baron, J. (2016). Foreword. In S. Li, 决策心理齐当别之 道. [Equate-to-differentiate way of decision-making] (p. 4). Shanghai, China: East China Normal University Press.

Böckenholt, U., \& Hynan, L.S. (1994). Caveats on a processtracing measure and a remedy. Journal of Behavioral Decision Making, 7, 103-117.

Brandstätter, E., Gigerenzer, G., \& Hertwig, R. (2006). The priority heuristic: Making choices without trade-offs. Psychological Review, 113, 409-432.

Brandstätter, E., \& Gussmack, M. (2012). The cognitive processes underlying risky choice. Journal of Behavioral Decision Making, 26, 185-197.

Busse, C., Kach, A.P., \& Wagner, S.M. (2017). Boundary conditions: What they are, how to explore them, why we need them, and when to consider them. Organizational Research Methods, 20, 574-609. 
Colbert, G., Murray, D., \& Nieschwietz, R. (2009). The use of expected value in pricing judgments. Journal of Risk Research, 12, 199-208.

DeKay, M.L., Hershey, J.C., Spranca, M.D., Ubel, P.A., \& Asch, D.A. (2006). Are medical treatments for individuals and groups like single-play and multiple-play gambles? Judgment and Decision Making, 1, 134-145.

Dshemuchadse, M., Scherbaum, S., \& Goschke, T. (2013). How decisions emerge: Action dynamics in intertemporal decision making. Journal of Experimental Psychology: General, 142, 93-100.

Fennema, H., \& Wakker, P. (1997). Original and cumulative prospect theory: A discussion of empirical differences. Journal of Behavioral Decision Making, 10, 53-64.

Fennema, H., \& Wakker, P. (1994). An explanation and characterization for the buying of lotteries. In R. Sixto (Ed.), Decision theory and decision analysis: Trends and challenges (pp. 163-175). Netherlands: Springer.

Gbadamosi, J., \& Zangemeister, W.H. (2001). Visual imagery in hemianopic patients. Journal of Cognitive Neuroscience, 13, 855-866.

Gigerenzer, G., Hertwig, R., \& Pachur, T. (2011). Heuristics: The foundations of adaptive behavior. New York, NY: Oxford University Press.

Glöckner, A., \& Herbold, A.K. (2011). An eye-tracking study on information processing in risky decisions: Evidence for compensatory strategies based on automatic processes. Journal of Behavioral Decision Making, 24, 71-98.

Grant, S., \& Kajii, A. (1998). AUSI expected utility: An anticipated utility theory of relative disappointment aversion. Journal of Economic Behavior \& Organization, 37, 277-290.

Hechanova, M.R.M., Waelde, L.C., \& Ramos, P.A.P. (2016). Evaluation of a group-based resilience intervention for typhoon haiyan survivors. Journal of Pacific Rim Psychology, 10, e12.

Hogarth, R.M. (1987). Judgement and choice: The psychology of decision. New York, NY: Wiley.

Johnson, E.J., Schulte-Mecklenbeck, M., \& Willemsen, M.C. (2008). Process models deserve process data: Comment on Brandstätter, Gigerenzer, and Hertwig (2006). Psychological Review, 115, 263-273.

Kahneman, D., \& Tversky, A. (1979). Prospect theory: An analysis of decision under risk. Econometrica, 47, 263-291.

Keren, G. (1991). Additional tests of utility theory under unique and repeated conditions. Journal of Behavioral Decision Making, 4, 297-304.

Klos, A., Weber, E.U., \& Weber, M. (2005). Investment decisions and time horizon: Risk perception and risk behavior in repeated gambles. Management Science, 51, 1777-1790.

Langer, T., \& Weber, M. (2001). Prospect theory, mental accounting, and differences in aggregated and segregated evaluation of lottery portfolios. Management Science, 47, 716-733.

Li, S. (1993). What is wrong with Allais' certainty effect? Journal of Behavioral Decision Making, 6, 271-281.

$\mathrm{Li}, \mathrm{S}$. (1994a). What is the role of transparency in cancellation? Organizational Behavior and Human Decision Processes, 60, 353-366.
Li, S. (1994b). Is there a problem with preference reversals? Psychological Reports, 74, 675-679.

$\mathrm{Li}, \mathrm{S}$. (1995). Is there a decision weight $\pi$ ? Journal of Economic Behavior and Organization, 27, 453-463.

Li, S. (1998). Can the conditions governing the framing effect be determined? Journal of Economic Psychology, 19, 133153.

Li, S. (2001). 艾勒悖论: 另释 [Allais paradox: A behavioral explanation]. Acta Psychologica Sinica, 33, 176-181

$\mathrm{Li}, \mathrm{S}$. (2003). The role of expected value illustrated in decisionmaking under risk: Single-play vs. multiple-play. Journal of Risk Research, 6, 113-124.

Li, S. (2004). A behavioral choice model when computational ability matters. Applied Intelligence, 20, 147-163.

Li, S. (2004). Equate-to-differentiate approach: An application in binary choice under uncertainty. Central European Journal of Operations Research, 12, 269-294.

Li, S. (2006). Preference reversal: A new look at an old problem. Psychological Record, 56, 411-428.

Li, S. (2016). 决策心理齐当别之道. [An equate-to-differentiate way of decision-making]. Shanghai, China: East China Normal University Press.

Li, S., \& Adams, A.S. (1995). Is there something more important behind framing? Organizational Behavior and Human Decision Processes, 62, 216-219.

Li, S., \& Taplin, J.E. (2002). Examining whether there is a disjunction effect in prisoner's dilemma games. Chinese Journal of Psychology, 44, 25-46.

Li, S., Taplin, J.E., \& Zhang, Y. (2007). The equate-todifferentiate's way of seeing the prisoner's dilemma. Information Sciences, 177, 1395-1412.

Li, S., \& Xie, X. (2006). A new look at the 'Asian disease' problem: A choice between the best possible outcomes or between the worst possible outcomes? Thinking and Reasoning, 12, 129143.

Li, X., Logan, G.D., \& Zbrodoff, N.J. (2010). Where do we look when we count? The role of eye movements in enumeration. Attention, Perception, \& Psychophysics, 72, 409-426.

Liang, Z.-Y., Xu, L.-J., Rao, L.-L., Jiang, T.-Z., \& Li, S. (2012). '20\%的概率获得蛋糕'=(获得蛋糕的 $20 \%$ '检验风险决策 的期望法则假设' ['20\% probability to gain a cake' = 'gain $20 \%$ of the cake'? Testing the expectation rule of risky decision making]. Chinese Science Bulletin, 57, 3421-3433.

Liu, H.-Z., Li, X., Li, S., \& Rao, L.-L. (2017). When expectation theories work or do not work: An eye-tracking study of discrepancy between everyone and every one. Manuscript submitted for publication.

Nalipay, M.J.N., \& Mordeno, I.G. (2016). Emotional intensity of trauma memory as moderator of the relationship between posttraumatic cognitions and PTSD symptoms. Journal of Pacific Rim Psychology, 10, e7.

Noton, D., \& Stark, L. (1971). Scanpaths in eye movements during pattern perception. Science, 171, 308-311.

Pachur, T., Hertwig, R., Gigerenzer, G., \& Brandstätter, E. (2013). Testing process predictions of models of risky choice: A 
quantitative model comparison approach. Frontiers in Psychology, 4, 646.

Paton, D. (2009). Living on the ring of fire: Perspectives on managing natural hazard risk in pacific rim countries. Journal of Pacific Rim Psychology, 3, 1-3.

Paton, D., Houghton, B.F., Gregg, C.E., McIvor, D., Johnston, D.M., Bürgelt, P., ... Horan, J. (2009). Managing tsunami risk: Social context influences on preparedness. Journal of Pacific Rim Psychology, 3, 27-37.

Quiggin, J. (1982). A theory of anticipated utility. Journal of Economic Behaviour \& Organizations, 3, 323-343.

Rao, L.-L., Li, S., Jiang, T., \& Zhou, Y. (2012). Is payoff necessarily weighted by probability when making a risky choice? Evidence from functional connectivity analysis. PLoS One, 7, e41048.

Rao, L.-L., Liang, Z.-Y., Li, S. (2009). 迫选规则体验法: 检验规 范性和描述性风险决策理论的新尝试 [The experiencing of imposed rule: A new attempt to test both normative and descriptive decision theories]. Acta Psychologica Sinica, 41, 726-736.

Rao, L.-L., Liu, X.-N., Li, Q., Zhou, Y., Liang, Z.-Y., Sun, H.-Y., ... Li, S. (2013). Toward a mental arithmetic process in risky choices. Brain and Cognition, 83, 307-314.

Rao, L.-L., Zhou, Y., Xu, L., Liang, Z.-Y., Jiang, T., \& Li, S. (2011). Are risky choices actually guided by a compensatory process? New insights from fMRI. PLoS One, 6, e14756.

Redelmeier, D.A., \& Tversky, A. (1992). On the framing of multiple prospects. Psychological Science, 3, 191-193.

Sagala, S., Okada, N., \& Paton, D. (2009). Predictors of intention to prepare for volcanic risks in Mt Merapi, Indonesia. Journal of Pacific Rim Psychology, 3, 47-54.

Scarpa, R., Zanoli, R., Bruschi, V., \& Naspetti, S. (2013). Inferred and stated attribute non-attendance in food choice experiments. American Journal of Agricultural Economics, 95, 165-180.

Schulte-Mecklenbeck, M., Kühberger, A., \& Ranyard, R. (2010). A handbook of process tracing methods for decision research: A critical review and user's guide. New York, NY: Taylor \& Francis.

Schulte-Mecklenbeck, M., Kühberger, A., Gagl, B., \& Hutzler, F. (2017). Inducing cognitive processes: Bringing process measures and cognitive processes closer together. Journal of Behavioral Decision Making, 30, 1001-1013.

Seiuli, B.M.S., Nikora, L.W., Te Awekotuku, N., \& Hodgetts, D. (2016). Fia ola: Grief recovery following a tsunami disaster in Samoa. Journal of Pacific Rim Psychology, 10, e5.
Su, Y., Rao, L.-L., Sun, H.-Y., Du, X.-L., Li, X., \& Li, S. (2013). Is making a risky choice based on a weighting and adding process? An eye-tracking investigation. Journal of Experimental Psychology: Learning, Memory, and Cognition, 3, 17651780.

Sun, H.-Y., Rao, L.-L., Zhou, K., \& Li, S. (2013). Formulationg an emergency plan based on expectation-maximization is one thing, but applying it to a single case is another. Journal of Risk Research, 17, 785-814.

Sun, Y., Li, S., Bonini, N., \& Su, Y. (2012). Graph framing effects in decision making. Journal of Behavioral Decision Making, 25, 491-501.

Takemura, K. (1994). Framing kouka no rironteki setumei: Risk ka ni okeru ishikettei no jyokyou-izonteki-syouten-model [Theoretical explanation of framing effect: Contingent focus model of decision framing]. Japanese Psychological Review, 37, 270-291.

Takemura, K., \& Fujii, S. (1999). Contingent focus model of decision framing under risk. Technical Report, 67, 51-67.

Thorngate, W. (1980). Efficient decision heuristics. Behavioral Science, 25, 219-225.

Tversky, A., \& Kahneman, D. (1981). The framing of decisions and the psychology of choice. Science, 211, 453-458.

Tversky, A., \& Kahneman, D. (1992). Advances in prospect theory: Cumulative representation of uncertainty. Journal of Risk and Uncertainty, 5, 297-323.

Underwood, G., Humphrey, K., \& Foulsham, T. (2008). Knowledge-based patterns of remembering: Eye movement scanpaths reflect domain experience. HCI and Usability for Education and Work, 5298, 125-144.

Wang, Z.-J., \& Li, S. (2012). 对整合模型和占优启发式模型的 检验基于信息加工过程的眼动研究证据 [Tests of the integrative model and priority heuristic model from the point of view of choice process: Evidence from an eye-tracking study]. Acta Psychologica Sinica, 44, 179-198.

Weber, E.U., Hsee, C.K., \& Sokolowska, J. (1998). What folklore tells us about risk and risk taking: Cross-cultural comparisons of American, Chinese and German proverbs. Organizational Behavior and Human Decision Processes, 75, 170-186.

Wedell, D.H., \& Böckenholt, U. (1994). Contemplating single versus multiple encounters of a risky prospect. American Journal of Psychology, 107, 499-518.

Zhou, L., Zhang, Y.Y., Wang, Z.J., Rao, L.L., Wang, W., Li, S., ... Liang, Z.Y. (2016). A scanpath analysis of the risky decisionmaking process. Journal of Behavioral Decision Making, 29, 169-182. 